\title{
Fatal respiratory infection due to ST308 VIM-1-producing Pseudomonas aeruginosa in a lung transplant recipient: case report and review of the literature
}

M. Carugati ${ }^{1,2^{*}}$ (D, A. Piazza ${ }^{3}$, A. M. Peri ${ }^{1}$, L. Carianii ${ }^{4}$, M. Brilli $^{5}$, D. Girelli ${ }^{4}$, D. Di Carlo ${ }^{3}$, A. Gramegna ${ }^{6,7}$,

M. Pappalettera ${ }^{6}$, F. Comandatore ${ }^{3}$, G. Grasselli ${ }^{7,8}$, A. P. Cantù 9 , M. Arghittu ${ }^{10}$, A. Gori ${ }^{1,7,11}$, C. Bandi ${ }^{5}$, F. Blasi ${ }^{6,7}$,

A. Bandera ${ }^{1,7}$ and IFALT working group

\begin{abstract}
Background: Data regarding the prevalence of metallo- $\beta$-lactamases (MBLS) among Pseudomonas aeruginosa isolates in cystic fibrosis patients are scarce. Furthermore, there is limited knowledge on the effect of MBL production on patient outcomes. Here we describe a fatal respiratory infection due to $P$. aeruginosa producing VIMtype MBLs in a lung transplant recipient and the results of the subsequent epidemiological investigation.

Case presentation: $P$. aeruginosa isolates collected in the index patient and among patients temporally or spatially linked with the index patient were analyzed in terms of antibiotic susceptibility profile and MBL production. Wholegenome sequencing and phylogenetic reconstruction were also performed for all $P$. aeruginosa isolates producing VIM-type MBLs. A VIM-producing $P$. aeruginosa strain was identified in a lung biopsy of a lung transplant recipient with cystic fibrosis. The strain was VIM-1-producer and belonged to the ST308. Despite aggressive treatment, the transplant patient succumbed to the pulmonary infection due to the ST308 strain. A VIM-producing P. aeruginosa strain was also collected from the respiratory samples of a different cystic fibrosis patient attending the same cystic fibrosis center. This isolate harbored the blaVIM-2 gene and belonged to the clone ST175. This patient did not experience an adverse outcome.

Conclusions: This is the first description of a fatal infection due to $P$. aeruginosa producing VIM-type MBLs in a lung transplant recipient. The circulation of $P$. aeruginosa isolates harboring MBLs pose a substantial risk to the cystic fibrosis population due to the limited therapeutic options available and their spreading potential.
\end{abstract}

Keywords: Cystic fibrosis, Lung transplant, Pseudomonas aeruginosa, Metallo- $\beta$-lactamases, Case report

\footnotetext{
* Correspondence: ela.carugati@gmail.com

'Division of Infectious Diseases, Fondazione IRCCS Ca' Granda Ospedale

Maggiore Policlinico, Via Francesco Sforza 35, 20122 Milan, Italy

${ }^{2}$ Division of Infectious Diseases and International Health, Duke University, 181

Hanes House, 300 Trent Drive, Durham 27710, USA

Full list of author information is available at the end of the article
}

C C The Author(s). 2020 Open Access This article is licensed under a Creative Commons Attribution 4.0 International License, which permits use, sharing, adaptation, distribution and reproduction in any medium or format, as long as you give appropriate credit to the original author(s) and the source, provide a link to the Creative Commons licence, and indicate if changes were made. The images or other third party material in this article are included in the article's Creative Commons licence, unless indicated otherwise in a credit line to the material. If material is not included in the article's Creative Commons licence and your intended use is not permitted by statutory regulation or exceeds the permitted use, you will need to obtain permission directly from the copyright holder. To view a copy of this licence, visit http://creativecommons.org/licenses/by/4.0/ The Creative Commons Public Domain Dedication waiver (http://creativecommons.org/publicdomain/zero/1.0/) applies to the data made available in this article, unless otherwise stated in a credit line to the data. 


\section{Background}

Pseudomonas aeruginosa is a non-glucose fermenter Gram-negative rod, frequently encountered in the respiratory tract of cystic fibrosis patients. $P$. aeruginosa is characterized by a low natural antimicrobial susceptibility and by an outstanding ability for selecting and spreading antimicrobial resistance in vivo $[1,2]$.

In 2018 the European Centre for Disease Prevention and Control (ECDC) reported that $19.2 \%$ of the P. aeruginosa invasive isolates tested were resistant to two or more antimicrobial groups [3]. Higher rates of multidrug resistance were found in the cystic fibrosis population; when $P$. aeruginosa isolates collected from cystic fibrosis patients in Northern Europe over the period 2006-2012 were analysed, multi-drug resistance was detected in $61.4 \%$ of the isolates [4]. Carbapenem resistance was reported by ECDC in $17.2 \%$ of the strains tested, with large inter-country variations $(0.0 \%$ in Iceland, $\mathbf{1 5 . 8 \%}$ in Italy, and $55.1 \%$ in Romania) [3].

Carbapenem resistance in $P$. aeruginosa is mediated by several mechanisms including: i) intrinsic $P$. aeruginosa resistance, such as the expression of inducible AmpC cephalosporinase, which is associated with a reduced susceptibility to imipenem [5]; ii) acquired resistance through chromosomal gene mutations, such as the mutational inactivation or downregulation of the $\operatorname{OprD}$ porin, which drives imipenem resistance and decreases meropenem susceptibility. In addition, the overexpression of efflux pumps of $P$. aeruginosa plays a major role in mutation-driven resistance [6]; iii) horizontally acquired resistance, such as genes encoding carbapenemases. These genes are typically located in class 1 integrons inserted into transposable elements. Among carbapenemases, metallo- $\beta$-lactamases (MBLs) are the most prevalent in $P$. aeruginosa. While at least nine different types of acquired MBLs have been described, the VIM-type is among the most important for geographic dissemination [7]. Furthermore, the VIM-type has broader substrate specificities and a higher affinity for carbapenems compared to other MBLs [8].

Carbapenemase production, and specifically MBL production, is rarely documented in patients with cystic fibrosis. Mustafa and collaborators did not detect any carbapenemase production when analyzing $P$. aeruginosa isolates collected from cystic fibrosis patients in Northern Europe [4]. Furthermore, the impact of $P$. aeruginosa antimicrobial resistance on the outcome of lung transplant in cystic fibrosis patients is still controversial [9]. To the best of our knowledge, four cases of MBLs detection in cystic fibrosis patients have been reported in the literature. Of these, only two reported outcome information and none were characterized by an adverse outcome [10-13]. Here we describe a fatal infection due to VIM1 - producing $P$. aeruginosa in a cystic fibrosis patient following lung transplantation. We also detail the epidemiological investigation performed in our medical center after the isolation of $P$. aeruginosa producing VIM-type MBLs.

\section{Case presentation Methods}

$P$. aeruginosa isolates collected in the index patient and among patients linked with the index patient were analyzed. The analysis involved the following steps: i) identification of bacterial isolates and susceptibility profile; ii) detection of carbapenemase activity; iii) whole genome sequencing (WGS) and phylogenetic analysis. Details can be found in the Supplementary Materials.

\section{Case description}

A 29 year-old female with end-stage cystic fibrosis lung disease (FEV1 21\%, lung allocation score 47.6) underwent bilateral orthotopic lung transplant in 2018. Pretransplant respiratory cultures were positive for extensively-drug resistant mucoid and non-mucoid $P$. aeruginosa with reduced susceptibility to carbapenems. She was exposed to carbapenems approximately 12 months prior to transplant. On day 7 after transplant the patient developed a right pleural empyema, requiring a thoracic drainage and broad-spectrum antimicrobial therapy (amikacin and ceftazidime). Respiratory cultures collected on the same day grew extensively-drug resistant mucoid $P$. aeruginosa (isolate SK76). SK76 showed a reduced susceptibility to meropenem but results were negative for carbapenemase production (Table 1). On day 34 , due to worsening clinical conditions and progression of the infection in the right pleural cavity, the patient was transferred to the intensive care unit (ICU) and a right pneumonectomy was performed. Lung biopsy cultures collected on day 34 grew extensively-drug resistant $P$. aeruginosa, resistant to carbapenems (isolate SK77). Resistance to carbapenems was mediated by the production of blaVIM-1 carbapenemase (Fig. 1). Despite aggressive antimicrobial treatment (levofloxacin, highdose colistin and high-dose extended-infusion meropenem) and invasive life-support measures (venous-venous extracorporeal membrane oxygenation and continuous renal replacement therapy), the patient died on day 56.

Whole genome sequencing (WGS) analysis showed that isolates SK76 and SK77 belonged to two different and unrelated Sequence Types (STs): ST253 and ST308, respectively (Fig. 2). Furthermore, SK76 and SK77 harbored a different resistance genes content. SK76 was characterized by the following resistance determinants: $\operatorname{aadA7,}$ a ac (6')-Ib7, blaPDC-9, blaOXA-488, blaOXA20, sul1, and gyrA. On the contrary, the following resistance genes were detected in SK77: blaVIM-1, aadA7, blaPDC-7, blaPER-1, blaOXA-488, and sul1 (Fig. 1). 
Table 1 Antimicrobial susceptibility profiles of the P. aeruginosa isolates SK76, SK77, and SK78

\begin{tabular}{|c|c|c|c|c|c|c|}
\hline \multirow{3}{*}{$\overline{\text { Amikacin }}$} & \multicolumn{2}{|l|}{ SK76 } & \multicolumn{2}{|l|}{ SK77 } & \multicolumn{2}{|l|}{ SK78 } \\
\hline & \multicolumn{2}{|c|}{$\mathrm{MIC}(\mathrm{mcg} / \mathrm{ml})$} & \multicolumn{2}{|c|}{$\mathrm{MIC}(\mathrm{mcg} / \mathrm{ml})$} & \multicolumn{2}{|c|}{$\mathrm{MIC}(\mathrm{mcg} / \mathrm{ml})$} \\
\hline & 16 & 1 & $>16$ & $\mathrm{R}$ & 16 & 1 \\
\hline Gentamicin & $>4$ & $\mathrm{R}$ & $>4$ & $\mathrm{R}$ & $>4$ & $\mathrm{R}$ \\
\hline Tobramycin & $>4$ & $\mathrm{R}$ & $>4$ & $\mathrm{R}$ & $>4$ & $\mathrm{R}$ \\
\hline Ciprofloxacin & $>1$ & $\mathrm{R}$ & 0.5 & $S$ & $>1$ & $\mathrm{R}$ \\
\hline Levofloxacin & $>2$ & $\mathrm{R}$ & 1 & S & $>2$ & $\mathrm{R}$ \\
\hline Colistin & $\leq 2$ & $\mathrm{~S}$ & $\leq 2$ & S & $\leq 2$ & S \\
\hline Piperacillin/tazobactam & $\leq 8$ & S & $>16$ & $\mathrm{R}$ & $>16$ & $\mathrm{R}$ \\
\hline Piperacillin & $\leq 8$ & S & $>16$ & $\mathrm{R}$ & $>16$ & $\mathrm{R}$ \\
\hline Cefepime & $>8$ & $\mathrm{R}$ & $>8$ & $\mathrm{R}$ & $>8$ & $\mathrm{R}$ \\
\hline Ceftazidime & 4 & $\mathrm{~S}$ & $>32$ & $\mathrm{R}$ & $>32$ & $\mathrm{R}$ \\
\hline Ceftazidime/avibactam & $\leq 2$ & S & $>8$ & $\mathrm{R}$ & $>8$ & $\mathrm{R}$ \\
\hline Ceftolozane/tazobactam & $\leq 1$ & S & $>4$ & $\mathrm{R}$ & $>4$ & $\mathrm{R}$ \\
\hline Imipenem & $>8$ & $\mathrm{R}$ & $>8$ & $\mathrm{R}$ & $>8$ & $\mathrm{R}$ \\
\hline Meropenem & 8 & । & 32 & $\mathrm{R}$ & 8 & I \\
\hline Fosfomycin & $>128$ & $\mathrm{R}$ & 16 & S & 16 & S \\
\hline Aztreonam & $\leq 1$ & S & $>256$ & $\mathrm{R}$ & $>256$ & $\mathrm{R}$ \\
\hline VIM-type carbapenemase & neg & & pos & & pos & \\
\hline
\end{tabular}

The detection of VIM-type MBLs among a cystic fibrosis patient prompted an epidemiological investigation in our medical center. All the respiratory cultures of patients attending the adult and pediatric cystic fibrosis center in 2018 were screened for the presence of MBLs. Similarly, all the patients admitted to the intensive care unit at the same time the index patient was admitted were screened for the presence of MBLs-producing isolates. A blaVIM-2 P. aeruginosa isolate was detected in the respiratory cultures of a male patient admitted to our adult cystic fibrosis center in 2018 for fever and worsening respiratory function (isolate SK78). The past medical history of this patient was characterized by: i) chronic colonization with methicillin-susceptible Staphylococcus aureus, mucoid and non-mucoid $P$. aeruginosa; ii) three respiratory exacerbations requiring treatment with carbapenems in the 12 months prior to the isolation of SK78; iii) end-stage lung disease (FEV1 27\%). After the isolation of SK78, the patient was treated with high-dose extended-infusion meropenem and amikacin, without any reported adverse effects. Antimicrobial treatment led to fever remission and global clinical improvement. Twelve months after the isolation of SK78, the patient was still alive with a stable, although severe, pulmonary function (FEV1 27\%). Isolate SK78 was characterized by a reduced susceptibility to meropenem and by the following additional resistance genes: $a a c\left(6^{\prime}\right)-I b 7$, blaOXA-50, and gyrA (Table 1 and Fig. 1). SK78 belonged to the epidemic high-risk clone ST175 (Fig. 2). No other MBLsproducing isolates were identified by our epidemiological investigation.

\section{Discussion and conclusions}

To the best of our knowledge, this is the first description of a fatal infection due to $P$. aeruginosa producing VIMtype MBLs in a cystic fibrosis lung transplant recipient. Among the four cases of MBLs-producing $P$. aeruginosa infections in cystic fibrosis patients already available in the literature, only two reported outcome data and only one involved a transplant recipient [10-13].

Our description highlights several substantial issues. First, the broad spectrum of resistance of $P$. aeruginosa isolates producing MBLs and the limited therapeutic options available for their treatment. Second, the virulence of $P$. aeruginosa isolates producing MBLs and their impact on the patient outcomes. Third, the spreading potential of $P$. aeruginosa isolates producing MBLs. Fourth, the need for stringent and efficient antimicrobial stewardship and infection control policies in cystic fibrosis centers.

\section{Broad spectrum of resistance of $P$. aeruginosa isolates} producing MBLs and limited therapeutic options available for their treatment

The broad spectrum of resistance of $P$. aeruginosa isolates producing MBLs restricts therapeutic options to few molecules, such as colistin, fosfomycin, aztreonam, and cefiderocol [7]. Of these, cefiderocol is not yet clinically available. Furthermore, some of these molecules (e.g. colistin) are 


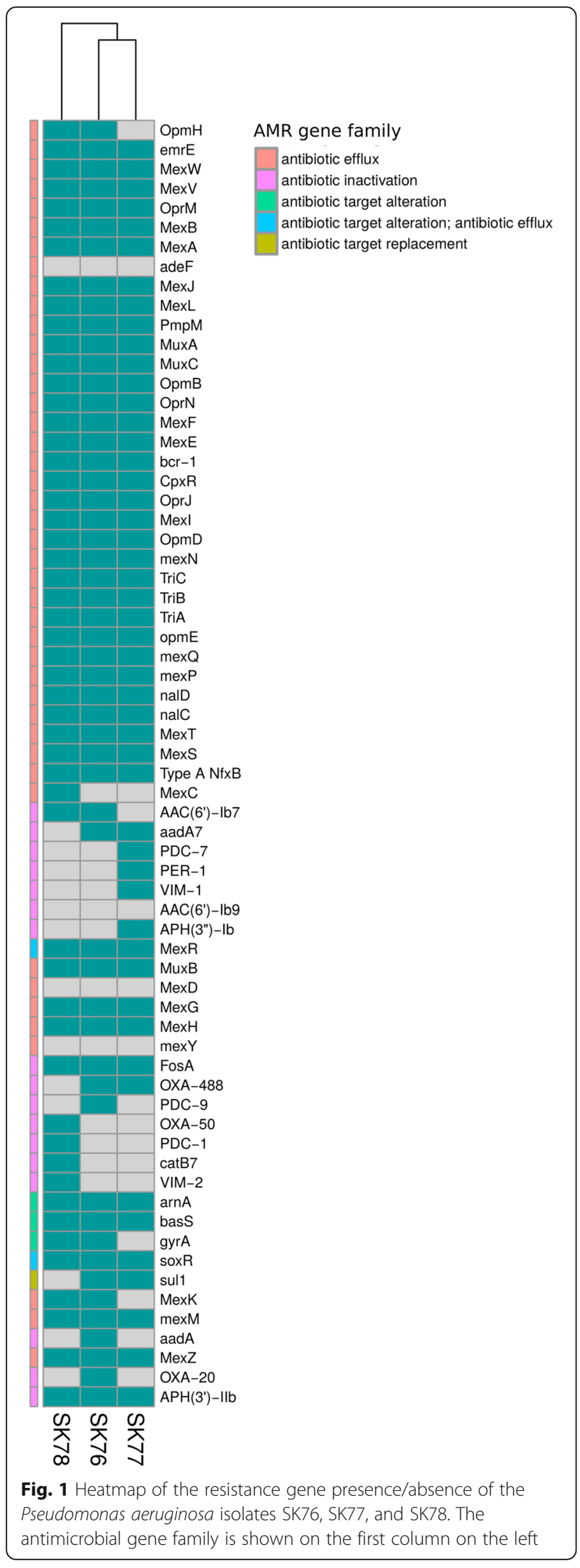

characterized by a narrow therapeutic window, which contributes to the occurrence of drug-related adverse events. It is also worth noting that the production of MBLs is typically associated with additional resistance mechanisms, as shown by strain SK77 and SK78. For this reason, combination therapies are needed to contain infections due to MBL-producing isolates. There is still no consensus on the most appropriate antibiotic combinations to be used in the setting of these difficult-to-treat infections [14].

\section{Virulence of $P$. aeruginosa isolates producing MBLs and their impact on the patient outcomes}

The evaluation of the virulence of $P$. aeruginosa isolates producing MBLs in comparison to the virulence of sensitive $P$. aeruginosa strains is a hot topic $[15,16]$. Acquisition of resistance is thought to be linked with fitness costs that decrease the virulence of multi-drug resistant $P$. aeruginosa strains [17-20]. However, several studies reported the presence of resistance mutations not associated with fitness costs and the development of compensatory or suppressor mutations in multi-drug resistant strains. Compensatory and suppressor mutations allow multi-drug resistant strains to regain their initial fitness, so that, in the end they preserve their original virulence $[19,21,22]$. Persoon and collaborators reviewed the charts of 198 patients admitted at the Erasmus Medical Center in Rotterdam (Netherlands) in the period 20082016 and who had a culture positive for $P$. aeruginosa producing blaVIM enzymes. $P$. aeruginosa producing blaVIM enzymes isolates were strongly associated with the death of 32 patients, leading to a $16.2 \%$ attributable mortality [23]. While the infection with strain SK77 likely contributed to the death of our female patient, the infection with strain SK78 did not substantially alter the clinical course of our male patient. Whether the adverse outcome of our female patient was mainly due to the pathogenicity of the VIM-producing isolate or to the severe pre-existing comorbidities of the host is difficult to be ascertained.

\section{Spreading potential of $P$. aeruginosa isolates producing MBLs}

MBLs are generally located on transposable genetic elements, which largely increases the spreading potential of these strains. Furthermore, ST308 is a recently reported emerging high-risk clone, while ST175 is among the three major international high-risk extensively-drug resistant clones and is widely distributed in several European countries [24]. Although isolates SK77 (ST308) and SK78 (ST175) did not cause an outbreak in our medical center, their spreading potential cannot be ignored. In this setting, appropriate epidemiological investigations and stringent infection control procedures are mandatory. 


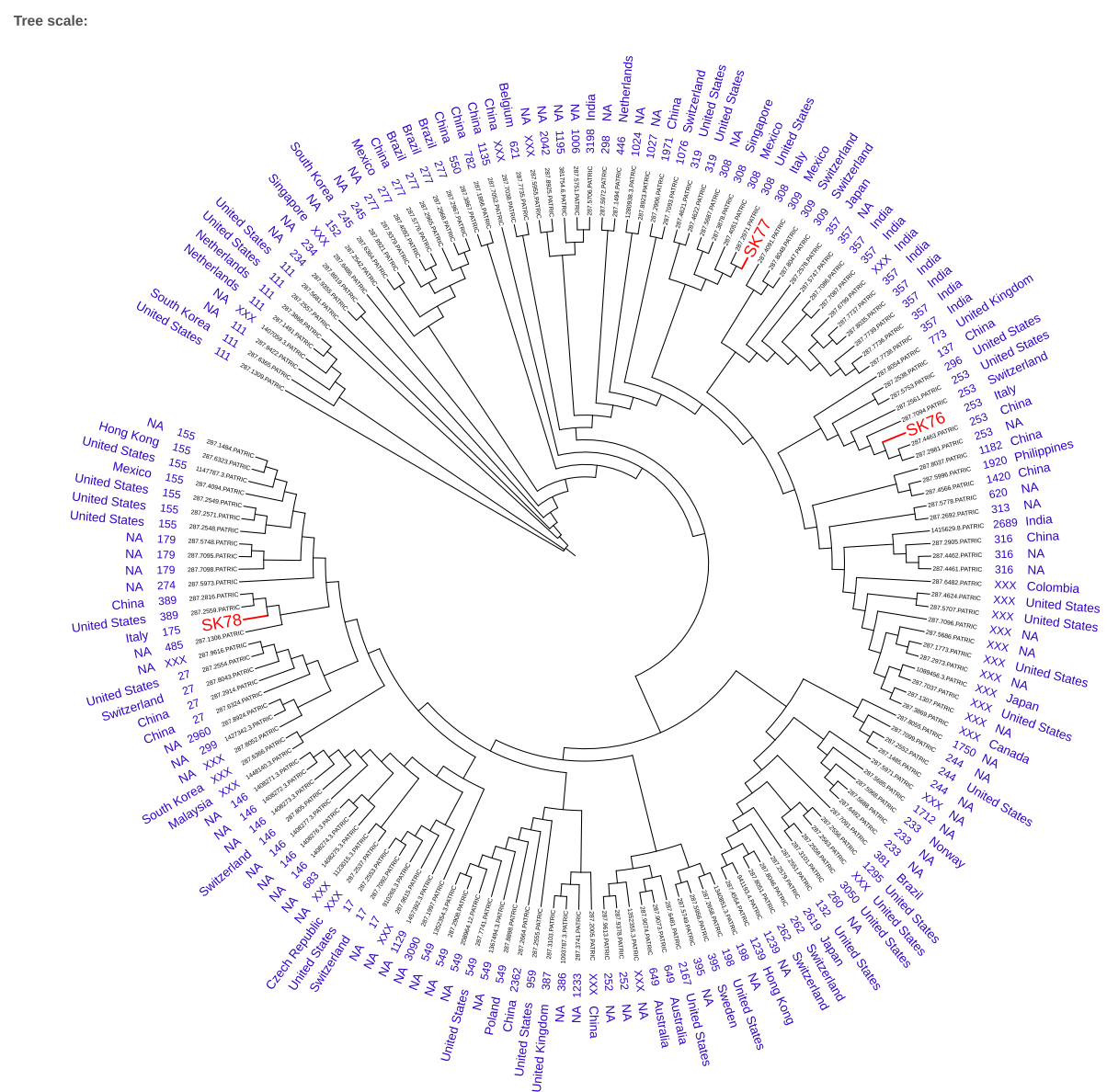

Fig. 2 Maximum likelihood phylogenetic tree including the three Pseudomonas aeruginosa isolates of the study (SK76, SK77 and SK78) and background strains retrieved from the PATRIC database

Need for stringent and efficient antimicrobial stewardship and infection control policies in cystic fibrosis centers

The selective antimicrobial pressure promoted by broadspectrum agents favors the emergence of resistant strains. Specifically, previous studies have shown an association between the use of piperacillin/tazobactam, quinolones, and cephalosporins and the emergence of VIM-positive $P$. aeruginosa isolates [25]. Furthermore, cystic fibrosis patients harboring MBLs in their respiratory tract may serve as hospital sources of carbapenemases. The acquisition of extensively-drug resistant $P$. aeruginosa isolates, especially clonal isolates, is known to be associated with increased pulmonary exacerbation rate, exaggerated lung function decline, and progression to end-stage lung disease [26]. For these reasons, segregation of patients harboring MBLs and environmental decontamination of areas where these patients transitioned may be need in order to avoid the dissemination of VIM-positive $P$. aeruginosa isolates in cystic fibrosis centers [13].

Despite the relevance of the data presented, this study has several limitations. We did not confirm the location of the blaVIM genes in class 1 integrons in the bacterial chromosome of the $P$. aeruginosa isolates evaluated. Genes encoding carbapenemases are generally found in class 1 integrons along with determinants of aminoglycoside resistance. These integrons are often inserted into transposable elements, which contributes to the spreading potential of these strains. Furthermore, we did not perform virulence studies. Our epidemiological investigation was restricted to the year 2018: the circulation of $P$. aeruginosa strains harboring MBLs in our medical center before 2018 was not assessed. Finally, our epidemiological investigation was limited to patients: potential environmental sources of $P$. aeruginosa isolates producing MBLs were not evaluated and the sources of the isolates SK77 and SK78 were not identified.

In summary, we described the first fatal infection due to $P$. aeruginosa producing VIM-type MBLs in a cystic fibrosis lung transplant recipient. $P$. aeruginosa producing VIM-type MBLs represents a matter of concern because of the limited therapeutic options available and its dissemination potential, especially in the setting of fragile hosts, such as cystic fibrosis patients. 


\section{Supplementary information}

Supplementary information accompanies this paper at https://doi.org/10. 1186/s12879-020-05338-3.

\section{Additional file 1}

\section{Abbreviations}

ECDC: European Centers for Disease Prevention and Control; FEV1: Forced expiratory volume in the first second; ICU: Intensive care unit; MBLS: Metallo$\beta$-lactamases; STs: Sequence types; WGS: Whole genome sequencing

\section{Acknowledgments}

We acknowledge the scientific contribution and support of the IFALT working group. Internal Medicine Department, Division of Infectious Diseases, Fondazione IRCCS Cà Granda Ospedale Maggiore Policlinico Milano (Milano, Italy): Laura Alagna, Marina Allegrini, Alessandra Bandera, Matteo Bolis, Manuela Carugati, Valentina Ferroni, Andrea Gori, Teresa Itri, Davide Mangioni, Debora Mondatore, Valeria Pastore, Federica Portunato. Internal Medicine Department, Respiratory Unit and Adult Cystic Fibrosis Center, Fondazione IRCCS Cà Granda Ospedale Maggiore Policlinico Milano (Milano, Italy): Stefano Aliberti, Francesco Blasi, Letizia Corinna Morlacchi, Martina Oriano, Valeria Rossetti, Paolo Tarsia, Leonardo Terranova. Thoracic Surgery and Lung Transplant Unit, Fondazione IRCCS Cà Granda Ospedale Maggiore Policlinico Milano (Milano, Italy): Rosaria Carrinola, Francesco Damarco, Paolo Mendogni, Mario Nosotti. Alessandro Palleschi, Ilaria Righi, Lorenzo Rosso, Davide Tosi. Pathology Department, Fondazione IRCCS Cà Granda Ospedale Maggiore Policlinico Milano (Milano, Italy): Stefano Bogetto Ferrero. Department of Department of Pathophysiology and Transplantation, Università degli Studi di Milano (Milano, Italy): Mario Clerici, Claudio Fenizia, Daria Trabattoni. Department of Oncology and Hemato-Oncology, Università degli Studi di Milano (Milano, Italy): Claudia Alteri, Carlo Federico Perno. Department of Clinical and Experimental Medicine, Università degli Studi di Sassari (Sassari, Italy): Giovanni Sotgiu. Genprobio srl: Marco Ventura, Claudio Pessina. Prossima Isola srl: Daniele Idini.

\section{Authors' contributions}

Conceptualization: M.C., A.M.P., C.B., F.B., A.G2., A.B. Methodology: M.C., A.M.P. L.C., A.P, A.B. Lab analysis: L.C, D.G., M.A., A.P., M.B., F.C., D.C.D. Writing, origina draft preparation: M.C., A.P., L.C, A.G1. Writing, review and editing: A.G1., M.P., G.G., A.P.C., IFALT working group. Supervision: C.B., F.B., A.G2., A.B. The author(s) read and approved the final manuscript.

\section{Funding}

This research was funded by the Italian 'Ministero dell'Istruzione, dell'Università e della Ricerca', grant number ARS01_00530. The funder had no role in the design of the study; in the collection, analyses, or interpretation of data; in the writing of the manuscript, or in the decision to publish the results.

\section{Availability of data and materials}

All data generated or analysed during this study are included in this published article.

\section{Ethics approval and consent to participate}

The submission of this case report for publication was notified to the local IRB (Fondazione IRCCS Ca' Granda Ospedale Maggiore Policlinico, Via Francesco Sforza 35, 20122 Milan, Italy) and the need for ethics approval was waived. The patient attending our adult cystic fibrosis center and in whom blaVIM-2 was detected signed written informed consent for participation in this case report.

\section{Consent for publication}

The patient attending our adult cystic fibrosis center and in whom blavIM-2 was detected signed written informed consent for publication in this study of his personal clinical details.

\section{Competing interests}

The authors declare that they have no competing interests.

\section{Author details}

'Division of Infectious Diseases, Fondazione IRCCS Ca' Granda Ospedale Maggiore Policlinico, Via Francesco Sforza 35, 20122 Milan, Italy. ${ }^{2}$ Division of Infectious Diseases and International Health, Duke University, 181 Hanes House, 300 Trent Drive, Durham 27710, USA. ${ }^{3}$ Romeo and Enrica Invernizzi Pediatric Research Center, Department of Biomedical and Clinical Sciences, University of Milan, Via Festa del Perdono 7, 20122 Milan, Italy. ${ }^{4}$ Cystic Fibrosis Microbiology Laboratory, Fondazione IRCCS Ca' Granda Ospedale Maggiore Policlinico, Via Francesco Sforza 35, 20122 Milan, Italy. ${ }^{5}$ Romeo and Enrica Invernizzi Pediatric CRC, Department of Biosciences, University of Milan, Via Festa del Perdono 7, 20122 Milan, Italy. ${ }^{6}$ Internal Medicine Department, Respiratory Unit and Adult Cystic Fibrosis Center, Fondazione IRCCS Cà Granda Ospedale Maggiore Policlinico, Via Francesco Sforza 35, 20122 Milan, Italy. 'Department of Pathophysiology and Transplantation, Università degli Studi di Milano, Via Festa del Perdono 7, 20122 Milan, Italy. ${ }^{8}$ Department of Anesthesia, Critical Care and Emergency, Fondazione IRCCS Cà Granda Ospedale Maggiore Policlinico, Via Francesco Sforza 35, 20122 Milan, Italy. ${ }^{9}$ Direzione Medica di Presidio, Fondazione IRCCS Cà Granda Ospedale Maggiore Policlinico, Via Francesco Sforza 35, 20122 Milan, Italy.

${ }^{10}$ Laboratory of Microbiology, Fondazione IRCCS Ca' Granda Ospedale Maggiore Policlinico, Via Francesco Sforza 35, 20122 Milan, Italy. ${ }^{11}$ Centre for Multidisciplinary Research in Health Science (MACH), University of Milan, Via Festa del Perdono 7, 20122 Milan, Italy.

Received: 21 May 2020 Accepted: 11 August 2020

Published online: 26 August 2020

\section{References}

1. Poole K. Pseudomonas aeruginosa: resistance to the max. Front Microbiol. 2011;2:65.

2. Breidenstein EBM, de la Fuente C, Hancock R. Pseudomonas aeruginosa: all roads lead to resistance. Trends Microbiol. 2011;19:419-26.

3. European Centre for Disease Prevention and Control. Surveillance of antimicrobial resistance in Europe 2018. Stockholm: ECDC; 2019.

4. Mustafa MH, Chalhoub H, Denis O, Deplano A, Vergison A, RodriguezVillalobos $\mathrm{H}$, et al. Antimicrobial susceptibility of Pseudomonas aeruginosa isolated from cystic fibrosis patients in northern Europe. Antimicrob Agents Chemother. 2016;60:6735-41.

5. Horcajada JP, Montero M, Oliver A, Sorlí L, Luque S, Gómez-Zorrilla S, et al. Epidemiology and treatment of multidrug-resistant and extensively drugresistant Pseudomonas aeruginosa infections. Clin Microbiol Rev. 2019;32: e00031-19.

6. Hocquet D, Berthelot P, Roussel-Delvallez M, Favre R, Jeannot K, Bajolet O, et al. Pseudomonas aeruginosa may accumulate drug resistance mechanisms without losing its ability to cause bloodstream infections. Antimicrob Agents Chemother. 2007;51:3531-6.

7. Cornaglia G, Giamarellou H, Rossolini GM. Metallo B-lactamases: a last frontier for B-lactams? Lancet Infect Dis. 2011;11:381-93.

8. Docquier JD, Lamotte-Brasseur J, Galleni M, Amicosante G, Frere JM, Rossolini GM. On functional and structural heterogeneity of VIM-type metallo-ß-lactamases. J Antimicrob Chemother. 2003;51:251-66.

9. Griffith BP, Hardesty RL, Armitage JM, Hattlet BG, Keenan RJ, Paradis I. A decade of lung transplantation. Annals Surg. 1993;218:310-20.

10. Emaneini M, Kalantar-Neyestanaki D, Jabalameli L, Hashemi M, Beigverdi R, Jabalameli F. Molecular analysis and antimicrobial resistance pattern of distinct strains of Pseudomonas aeruginosa isolated from cystic fibrosis patients in Iran. Iran J Microbiol. 2019;11:98-107.

11. Cardoso O, Alves AF, Leitao R. Metallo-B-lactamase VIM-2 in Pseudomonas aeruginosa isolates from a cystic fibrosis patient. Int J Antimicrob Agents. 2008;31:375-9.

12. Pollini S, Fiscarelli E, Mugnaioli C, Di Pilato V, Ricciotti G, Neri AS, et al. Pseudomonas aeruginosa infection in cystic fibrosis caused by an epidemic metallo- $\beta$-lactamase-producing clone with a heterogeneous carbapenem resistance phenotype. Clin Microbiol Infect. 2011;17:1272-5.

13. Pollini S, Mugnaioli C, Dolce D, Campana S, Neri AS, Taccetti G, et al. Chronic infection sustained by a Pseudomonas aeruginosa high-risk clone producing the VIM-1 metallo- $\beta$-lactamase in a cystic fibrosis patient after lung transplantation. J Cyst Fibros. 2018;17:470-4.

14. Petrosillo N, loannidou E, Falagas ME. Colistin monotherapy vs. combination therapy: evidence from microbiological, animal and clinical studies. Clin Microbiol Infect. 2008;14:816-27. 
15. Juan C, Pena J, Oliver A. Host and pathogen biomarkers for severe Pseudomonas aeruginosa infections. J Infect Dis. 2017;215:S44-51.

16. Gomez-Zorrilla S, Juan C, Cabot G, Camoez M, Tubau F, Oliver A, et al. Impact of multidrug resistance on the pathogenicity of Pseudomonas aeruginosa: in vitro and in vivo studies. Int J Antimicrob Agents. 2016;47:368-74.

17. Andersson DI. The biological cost of mutational antibiotic resistance: any practical conclusions? Curr Opin Microbiol. 2006;9:461-5.

18. Andersson $\mathrm{DI}$, Hughes D. Antibiotic resistance and its cost: is it possible to reverse resistance? Nat Rev Microbiol. 2010;8:260-71.

19. Olivares Pacheco J, Alvarez-Ortega C, Alcalde Rico M, Martinez JL. Metabolic compensation of fitness costs is a general outcome for antibiotic-resistant Pseudomonas aeruginosa mutants overexpressing efflux pumps. mBio. 2017; 8:e00500-17.

20. Sun Z, Jiao X, Peng Q, Jiang F, Huang Y, Zhang J, et al. Antibiotic resistance in Pseudomonas aeruginosa is associated with decreased fitness. Cell Physiol Biochem. 2013;31:347-54.

21. Skurnik D, Roux D, Cattoir V, Danilchanka O, Lu X, Yoder-Himes DR, et al. Enhanced in vivo fitness of carbapenem-resistant oprD mutants of Pseudomonas aeruginosa revealed through high-throughput sequencing. Proc Natl Acad Sci U S A. 2013;110:20747-52.

22. Suarez C, Pena C, Gavald L, Tubau F, Manzur A, Dominguez MA, et al. Influence of carbapenem resistance on mortality and the dynamics of mortality in Pseudomonas aeruginosa bloodstream infection. Int J Infect Dis. 2010;14:e73-8.

23. Persoon MC, Voor AF, van Meer MPA, Bokhoven KC, Gommers D, Vos MC, et al. Mortality related to Verona Integron encoded Metallo- $\beta$-lactamasepositive Pseudomonas aeruginosa: assessment by a novel clinical tool. Antimicrob Resist Infect Control. 2019;8:107.

24. Abdouchakour F, Aujoulat F, Licznar-Fajardo P, Marchandin H, Toubiana M, Parer $\mathrm{S}$, et al. Intraclonal variations of resistance and phenotype in Pseudomonas aeruginosa epidemic high-risk clone ST308: a key to success within a hospital? Inter J Med Microbiol. 2018;308:279-89.

25. Voor AF, Severin JA, Hagenaars MBH, de Goeij I, Gommers D, Vos MC. VIMpositive Pseudomonas aeruginosa in a large tertiary care hospital: matched case control studies and a network analysis. Antimicrob Resist Infect Control. 2018;7:32

26. Parkins MD, Somayaji R, Waters VJ. Epidemiology, biology, and impact of clonal Pseudomonas aeruginosa infections in cystic fibrosis. Clin Microbiol Rev. 2018;31:e00019.

\section{Publisher's Note}

Springer Nature remains neutral with regard to jurisdictional claims in published maps and institutional affiliations.

Ready to submit your research? Choose BMC and benefit from:

- fast, convenient online submission

- thorough peer review by experienced researchers in your field

- rapid publication on acceptance

- support for research data, including large and complex data types

- gold Open Access which fosters wider collaboration and increased citations

- maximum visibility for your research: over $100 \mathrm{M}$ website views per year

At $\mathrm{BMC}$, research is always in progress.

Learn more biomedcentral.com/submissions 